\title{
Does it matter who does ultrasound examination of the gall bladder?
}

\author{
TIMOTHY G ALLEN-MERSH， ROGER W MOTSON， WILLIAM HATELY
}

The high level of accuracy achieved by some ultrasonographers in the diagnosis of gall stones ${ }^{12}$ has resulted in a general acceptance of ultrasound as the primary investigation for gall stones. ${ }^{3}$ These excellent results, however, were obtained by strongly motivated and highly experienced ultrasonographers conducting clinical trials. ${ }^{4}$ The knowledge that the result of an ultrasound examination will be included in a prospective study may improve performance. We have sought to establish whether the same level of accuracy can be achieved when ultrasound is performed outside the confines of a prospective trial by ultrasonographers with varying degrees of experience.

The purposes of this study were, firstly, to assess the performance of gall bladder ultrasound in a busy radiology department where gall bladder ultrasound is routinely performed by different radiologists and, secondly, to see whether there was "operator variability" between the ultrasonographers performing the scans. The results were examined retrospectively and represent a record of what occurred in our department without the possible bias which would be introduced by a prospective study.

\section{Methods and results}

We analysed the results of 402 ultrasound examinations of the gall bladder performed on a Sonotron Diasonic DS1 real time scanner, using a $3.5 \mathrm{mHz}$ probe, during a two year period up to June 1984. The scans were performed by four consultant radiologists (operators 1-4) and various registrars and

The London Hospital, Whitechapel, London E1 1BB

TIMOTHY G ALLEN-MERSH, MD, FRCS, senior surgical registrar

ROGER W MOTSON, MS, FRCS, senior surgical registrar

WILLIAM HATELY, FRCPED, FRCR, consultant radiologist

Correspondence to: Mr R W Motson, Consultant Surgeon, Colchester General Hospital, Colchester, Essex CO4 5JL. senior registrars (grouped together as operator 5). A total of $174(43 \%)$ of the scans were not accompanied by other gall bladder investigations or by surgery, either because the scan did not show gall stones and the subsequent history did not suggest recurring symptoms of gall stones (160 cases) or because the scan suggested stones but operation was inappropriate (14 cases). A deliberate bias in favour of investigation by ultrasound was adopted by including these results in the study and assuming that all of the scans were correct.

Scans were categorised into the following five groups.

False positive scans were those reported as showing gall stones when stones were not subsequently found in the gall bladder at operation.

False negative scans were those reported as showing no gall stones when stones were detected by oral cholecystography, intravenous cholangiography, or endoscopic retrograde cholangiopancreatography and subsequently found at operation.

Equivocal scans were those where the gall bladder could not be visualised or where gall stones were suspected but a confirmatory oral cholecystogram was advised.

Correct diagnosis of stones-These were scans reported as showing gall ${ }^{*}$ stones where stones were confirmed at operation or necropsy or by oral cholecystography, intravenous cholangiography, or endoscopic retrograde cholangiopancreatography; scans suggesting stones in patients with a normal oral cholecystogram who did not undergo surgery; and scans showing stones which were assumed to be correct (see above).

Correct exclusion of stones-These were scans reported as showing no gall

Number of ultrasound scans of gall bladder in each diagnostic category by ultrasound operator who performed scan

\begin{tabular}{ccccccc}
\hline & $\begin{array}{c}\text { Correct } \\
\text { diagnosis } \\
\text { op gall stones }\end{array}$ & $\begin{array}{c}\text { Correct } \\
\text { oxclusion } \\
\text { of gall stones }\end{array}$ & $\begin{array}{c}\text { False } \\
\text { positive }\end{array}$ & $\begin{array}{c}\text { False } \\
\text { negative }\end{array}$ & $\begin{array}{c}\% \\
\text { Incorrect }\end{array}$ & $\begin{array}{c}\text { No (\%) } \\
\text { equivocal }\end{array}$ \\
\hline 1 & 15 & 47 & 1 & 4 & 7 & 0 \\
2 & 23 & 58 & 0 & 2 & 2 & $13(14)$ \\
3 & 14 & 22 & 2 & 3 & 10 & $7(15)$ \\
4 & 6 & 30 & 0 & 0 & 0 & $2(5)$ \\
5 & 36 & 97 & 3 & 6 & 6 & $12(8)$ \\
\hline All & 94 & 254 & 6 & 15 & 5 & $34(8)$ \\
\hline
\end{tabular}

Variation significantly greater $(p=0 \cdot 01)$ than expected by chance was found in proportion of cases Variation significantly greater $(\mathrm{p}=0 \cdot 01)$ than expected by chance was found in proportion of cases
reported correctly by different operators. Wide variation was also found between operators in proportion of equivocal scans. 
stones, supported by findings at oral cholecystography, intravenous cholangiography, or endoscopic retrograde cholangiopancreatography; and scans reporting no stones which were assumed to be correct (see below).

The table shows the number of cases falling into each category for each ultrasonographer. The proportion of incorrect scans performed by registrars $(9 / 154 ; 6 \%)$ was similar to that by consultants $(12 / 248 ; 5 \%)$. Operators 2 and 4 both performed a significantly lower proportion of incorrect scans than operator $3\left(\chi^{2}\right.$ test, contingency table; $\left.p<0.05\right)$ : Operators 2 and 4 both performed a significantly lower $(p<0.05)$ proportion of incorrect scans than all other operators together.

There was wide variation $(0-15 \%)$ in the proportion of equivocal scans performed by different operators.

\section{Discussion}

Assessment of the performance of ultrasound is beset with difficulties, such as differing patient selection, comparability of operators, and validation of results. For a true comparison of operators the same patients would have to be examined by each ultrasonographer (more than five in our study) on the same equipment, since review of still images taken during a scan by another ultrasonographer is inadequate. Ideally, the patient group studied should be those patients with symptoms suggestive of gall stone disease who would otherwise have been investigated by oral cholecystography. The participating ultrasonographers should use identical criteria for the diagnosis and exclusion of stones. Validation of the scans could be achieved only by subjecting all patients to laparotomy soon after the ultrasound investigation. Plainly such a study would be impossible to perform. Although our study falls short of this ideal, it does identify some difficulties which may be associated with the widespread introduction of ultrasound examination of the gall bladder.

The assumptions made in this study erred in favour of the accuracy of ultrasound, but overall at least $5 \%$ of scans were misleading and $8 \%$ were non-contributory.

The proportion of incorrect scans varied from nil to $11 \%$ among different ultrasonographers. While there was no significant difference in the proportion of incorrect scans performed by consultants compared with registrars, there were significant differences between individual operators and between the two most accurate ultrasonographers and the other operators. There was also a wide variation $(0-15 \%)$ between operators in the proportion of equivocal scans. Ultrasonographers with an equivocal scan rate of more than $10 \%$ did not perform fewer inaccurate scans $(7 / 144 ; 5 \%)$ than operators with an equivocal scan rate of less than $10 \%(14 / 258$; $5 \%$ ). Thus cautious reporting did not lessen the number of inaccurate scans.

The most serious scanning error is the false positive result, since this may lead to unnecessary surgery. In this study no false positive scans were performed by the two most accurate ultrasonographers, but overall $6 \%$ of patients undergoing cholecystectomy for gall stones diagnosed by ultrasound did not have stones in the gall bladder at operation. An error of this order throughout England and Wales could result in over 2000 erroneous gall bladder explorations a year. ${ }^{5}$ Criteria may be laid down for the diagnosis and exclusion of stones on ultrasound scans. ${ }^{6}$ Strict application of these criteria may be an important way of reducing the false positive rate.

It could be argued that our study was biased as we included the 174 scans which were not followed by any further investigation. Since the false positive and equivocal scan results arose from the whole population studied, exclusion of the non-validated cases would have introduced a misleading high false positive and equivocal scan rate. Inclusion of the non-validated scans with the assumption that they were correct $(87 \%$ of the validated scans were correct) resulted in an error rate which was unlikely to be an overestimate. Since the proportion of non-validated scans was similar for each operator, this assumption did not bias comparisons between operators.

All the false positive scans were confirmed by operation, and while the false positive rate may be altered by the inclusion or exclusion of non-validated patients the absolute number of false positive scans remains unaltered.

We conclude that there does appear to be a wide variation between ultrasonographers both in the proportion of incorrect results-including false positive scans_-and in the threshold for reporting findings as equivocal. The widespread introduction of ultrasound for the diagnosis of gall stones may be expected to introduce an operator variation which will reduce the diagnostic accuracy of this technique.

We are greatly indebted to Mrs Brenda Nicholls, of the ultrasound department, London Hospital, for help with data retrieval and to Mrs Allison Shipton for typing the manuscript.

\section{References \\ 1 Cooperberg PL, Burhenne HJ. Realtime ultrasonography; diagnostic technique of choice in calculous gallbladder disease. $N$ Engl F Med 1980;302:1277-9. \\ 2 DeLacey G, Gajiar B, Twomey B, et al. Should cholecystography or ultrasound be the primary investigation for gallbladder disease? Lancet 1984;i:205-7. \\ 3 Bouchier IAD. Imaging procedures to diagnose gallbladder disease. Br Med $\mathcal{J} 1984$;288:1632-3. \\ 4 Anonymous. Echoes from the gallbladder [Editorial]. BrMed $\mathcal{f} 1981 ; 282: 3$. \\ 5 Department of Health and Social Security; Office of Population Censuses and Surveys; Welsh Office. Hospital in-patient enquiry. Main tables 1978. London: HMSO, 1981. (Series MB4, No 12.) \\ 6 Cosgrove DO, McCready R. Ultrasound imaging. Chichester: Wiley, 1982:238 \\ (Accepted 2 May 1985)}

WORDS GLUTEN and COLLA. The original glue (gluten in Latin) is now an almost obsolete adhesive made from animal hides, bones, and fish residues. It had a number of disadvantages in practice, being slow to melt for application and, after setting, vulnerable to heat and moisture. It has been superseded by a wide range of synthetic substances which are free from these disadvantages and are generally known as adhesives. Unfortunately, the volatile organic solvents in which they are applied have tempted mainly youngsters to inhale the solvents and induce thereby a state of quasiinebriation, a dangerous practice which has led to some deaths. However, the British penchant for monosyllables has brought back the obsolescent word glue, since the practice is known as GLUE SNIFFING. We also have GLUE EAR for chronic seromucous (secretory/catarrhal) otitis media.

When the starch is washed out of wheat flour, the residue is a sticky mass which is named GLUTEN, the Latin for glue. Indeed, starch paste is still a common adhesive. Gluten provides the elasticity which imparts coherence to the "rising" dough in bread making. Likewise, the specially high gluten content of the wheat variety Triticum durum induces the characteristic plasticity of pasta. Unfortunately, gluten induces coeliac disease in those who are endowed with that predisposition. GLUTAMIC ACID, an amino acid widely distributed in tissues, especially in the brain, and indirectly a neurotransmitter, is so called because of its high concentration in gluten. Monosodium GLUTAMATE adds that meaty taste which enables the gastro- nomic plebs to swallow with relish what would otherwise be tasteless.

The Greeks also had a word for glue-kolla. In its English language manifestations the $\mathrm{k}$ is changed to $\mathrm{c}$. COLLAGEN, the fibrous protein that holds the body together, is so called from the glue to which it is converted on boiling (kolla+genes, to become). Collord was so called from the gummy consistency of the early recognised organic colloids, though the term has now been widened to comprise also a range of non-sticky varieties and the present definition is based on molecular structure. COLLODION, another gluey substance, is a solution of gun cotton (cellulose nitrate) in ether and alcohol. Applied in the manner of a paint, it is used to fix small dressings and splints for digital injuries.

No research project may be launched without a statement of PROTOCOL. Originally a protocol was a flyleaf glued to the case of a volume, and containing an account of its contents (Gk protōs, first $+k o l l a$, glue). Over the past four centuries it has left its gluey origin and undergone several shifts and diversifications of meaning - travelling via the original note of a transaction; then the original draft of a diplomatic document, especially one of the propositions agreed to and signed by the parties to be embodied in a formal treaty; to the current medical usage as a precise and detailed statement of the plan for a research project. COLLAGE is a picture made up from scraps of paper and various odds and ends glued to the canvas. It is not an old lady's choker.-B J FREEDMAN. 